REVIEW

\title{
Alternative Splicing and Its Impact as a Cancer Diagnostic Marker
}

\author{
Yun-Ji Kim, Heui-Soo Kim* \\ Department of Biological Sciences, College of Natural Sciences, Pusan National University, Busan 609-735, Korea
}

\begin{abstract}
Most genes are processed by alternative splicing for gene expression, resulting in the complexity of the transcriptome in eukaryotes. It allows a limited number of genes to encode various proteins with intricate functions. Alternative splicing is regulated by genetic mutations in cis-regulatory factors and epigenetic events. Furthermore, splicing events occur differently according to cell type, developmental stage, and various diseases, including cancer. Genome instability and flexible proteomes by alternative splicing could affect cancer cells to grow and survive, leading to metastasis. Cancer cells that are transformed by aberrant and uncontrolled mechanisms could produce alternative splicing to maintain and spread them continuously. Splicing variants in various cancers represent crucial roles for tumorigenesis. Taken together, the identification of alternative spliced variants as biomarkers to distinguish between normal and cancer cells could cast light on tumorigenesis.
\end{abstract}

Keywords: alternative splicing, biological markers, neoplasms, neoplastic cell transformation

\section{Introduction}

Genes are transcribed into pre-mRNA and pass through splicing as a post-transcriptional modification to generate mature mRNA for translation [1]. Splicing is an essential process for gene expression in eukaryotes, eliminating introns and joining the exons, which occurs in the spliceosome, a complex of small nuclear ribonucleoproteins (snRNPs) and other proteins [2]. This splicing can generate alternative spliced transcripts from pre-mRNA and different exon constitutions, resulting in different proteins. Alternative splicing can occur in different ways. The most representative mechanisms are as follows: 1, extending or shortening the exon by alternative donor and acceptor sites; 2, exon skipping; 3, mutual exon exclusion; and 4, intron retention $[1,3,4]$. These mechanisms allow protein isoforms with different biological characters to be produced from single genes [1]. Therefore, complex transcriptomes and proteomes could be derived from a limited number of genes [4]. Namely, this process is an important strategy for the complicated regulation of eukaryotes, and most genes (92-95\%) undergo this process $[5,6]$.
Alternative splicing is regulated according to cell type, developmental stage, and disease states $[1,7,8]$. Biochemical mechanisms for the recognition of splice sites are not understood clearly according to cellular conditions, but some tissue-specific factors participate in alternative splicing [4]. In addition, quantitative gene expression is controlled by nonsense-mediated decay mechanisms that degrade targeting mRNAs, producing nonsense mutations. Thus, truncated or erroneous proteins with abnormal functions are prevented from being expressed [9]. Alternative transcripts could be related to various diseases, including cancer. As many as 50\% of genetic diseases of humans are related to mutations in splice site sequences and regulatory elements, such enhancers and silencers, resulting in alternative exon constitution $[3,10,11]$. Recently, the SpliceDisease database, providing information for relationships among gene mutation, splicing defects, and diseases, was reported [12]. Especially, aberrent spliced variants are found frequently in cancer, indicating that they could play a role for the survival of cancer cells [8]. Alternative splicing of cancer-related genes could affect cell cycle control, signal transduction pathways, apoptosis, angiogenesis, invasion, 
and metastasis $[8,13]$.

Cancer markers allow us to determine the prognosis and therapy for cancer during the remedy of cancer. Thus, the identification of cancer markers is highlighted in the cancer research field [14]. Cancers result from the accumulation of complex genetic and epigenetic alterations against normal regulation. Cancerous cells grow irregularly, create malignant tumors, and move to the other parts of the body. Their alternative spliced transcripts could be detected with cryptic splicing sites. Accordingly, alternative transcripts produced by splicing events represent good candidates for cancer biomarkers $[7,8,13]$. In the present review, we summarize and discuss the alternative splicing events and their potential as cancer biomarkers.

\section{Genetic and Epigenetic Regulation of Alternative Splicing}

Alternative splicing is an essential post-transcriptional process for creating various protein isoforms from the same gene. Approximately $60 \%$ of human genes have at least one alternative splice transcript [15]. Alternative splicing is regulated by mutations in genetic regions $[3,7,14]$. Most exons are flanked by the intronic dinucleotides GT (donor site) and AG (acceptor site), which are recognized by the spliceosome (Fig. 1A). Pre-mRNA is reconstructed only by joined exons with the removal of introns. However, mutations of these splice sites lead to aberrant splicing, producing exon skipping and different joining of exons to truncated or nonfunctional or dysfunctional proteins. It was reported that mutations of splice sites may play important roles in human disease, and longer proteins also tend to associate to various disease $[16,17]$.

In the case of the PAX6 gene, a 5' splice site mutation in intron 12 induces exon skipping in relation to autosomal dominant aniridia [18]. In the APC gene, abnormal splicing by a 3' splice site mutation in intron 3 causes exon 4 skipping due to a frameshift in hepatoblastoma [19]. A mutation in intron 25 of the $A B C A 3$ gene creates novel 5 ' splice sites [20]. The spliceosome is known to recognize cryptic splice sites instead of typical splice sites. Noncanonical splice sites are GC-AG, GG-AG, GT-TG, GT-CG, AT-AG, GA-AG, GT- AC, and CT-AG (5'-3' splice sites) [21-23]. Mutations at the noncanonical splice site of the SEDL gene cause variants in X-linked spondyloepiphyseal dysplasia tarda [24]. Additionally, less exon skipping and more intron retention by

(A) Genetic regulation

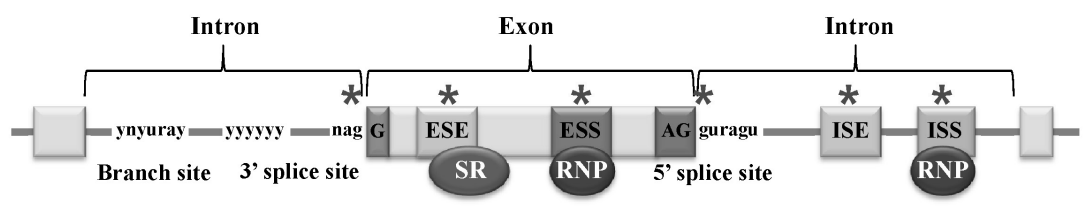

(B) Epigenetic regulation

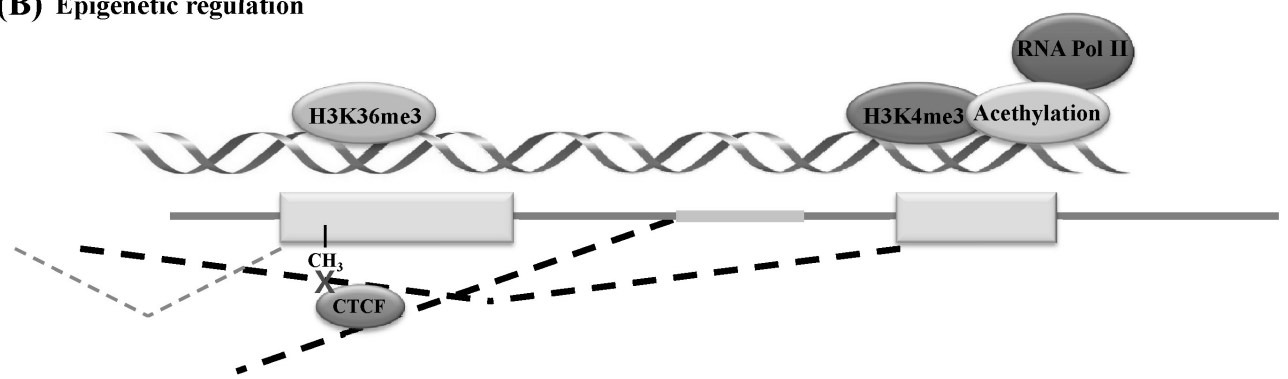

Fig. 1. Genetic and epigenetic regulation of alternative splicing. (A) Most genes are spliced by typical splice sites (GT-AG) and regulatory sequences in exons (ESS and ESE) and introns (ISS and ISE). Mutations $\left({ }^{*}\right)$ generate alternative splicing, resulting in various transcripts from a single gene. (B) H3K36me3 marking in exons is found in weakly expressed alternatively spliced exons (grey thin dotted line), and H3K4me3 marks are rich in spliced exons (black thick dotted line), indicating that histone modification has a relation to transcription via splicing-related marking mechanisms. Additionally, hisone acethylation could modulate splicing rates to react quickly to changing conditions with increased RNA polymerase II processivity, and spliceosome rearrangements are affected by histone acetylation. High levels of CG have a tendency not to be spliced (grey line in intron), and exon skipping is in the lower level of both CG and mCG in exonic regions. Methylation of exon inhibits binding of CCCTC-binding factor (CTCF), leading to exon skipping. ESE, exonic splicing enhancers; ISE, intronic splicing enhancers; ESS, exonic and intronic splicing silencers; ISS, intronic splicing silencers; SR, arginine-serine-rich; RNP, ribonucleoprotein. 
alternative splice sites are observed in cancer tissue compared to normal tissues from an analysis of expression sequence tags (ESTs) [25]. As a mutation of pre-mRNA, A to I RNA editing (conversion adenosine to inosine from deamination) also affects transcriptome diversification. RNA editing has regulatory roles, such as altering splice sites and sequences necessary for recognition of the spliceosome, resulting in modulation of alternatively spliced transcripts [26]. The last case of alternative splicing is by cis-genomic mutations in regulatory factors, such as branch sites, exonic and intronic splicing enhancers, and silencers [7, 27]. PremRNA has exonic and intronic splicing enhancers (ESEs, ISEs) and silencers (ESSs, ISSs) that promote exon inclusion and exclusion by regulation of splice site recognition, respectively (Fig. 1A). The arginine-serine-rich (SR) protein that binds to ESEs induces splicing via a helping assembly spliceosome by interacting with snRNP. On the contrary, heterogeneous nuclear ribonucleoprotein is bound to ESEs and ISEs and inhibits splice site recognition by blocking spliceosome assembly [15, 27-29]. Mutations in regulatory factor could disturb the binding of these spliceosome assembly-related proteins. Mutations at nucleotide positions 57 and 58 of the 174-bp-long exon 7 cause exon 7 skipping as a result of aberrant splicing by interrupted ESE-specific consensus sequences that are recognized by the SC35 and SF2/ASF SR proteins [10, 30]. Additionally, Ron, encoding the tyrosine kinase receptor for macrophage-stimulating protein, has alternative splice transcripts. It is regulated by overexpression of SF2/ASF binding to ESE and ISE in colon and breast cancer [31].

Alternative splicing is also known to be affected by epigenetic regulation, such as DNA methylation, histone modification, and chromatin structure (Fig. 1B). The relationship between chromatin structure and alternative splicing is still in a maze, but association studies are gradually increasing genomewide [32-34]. Hisone modifications are enriched in exons rather than introns and related to exon expression, especially H3K36me3, H3K79me1, H2BK5me1, H3K27me1, H3K27me2, and H3K27me3 [35, 36]. H3K36me3 marking in exons is found in weakly expressed, alternatively spliced exons, indicating that histone modification has a relation to transcription via splicing-related marking mechanisms [36, 37]. These histone marks could recruit splicing regulators with chromatin binding proteins and affect mRNA splicing [33]. Additionally, hisone acethylation could modulate splicing rates to react quickly to changing conditions with increased RNA polymerase II processivity, and spliceosome rearrangements are affected by histone acetylation [38]. Nevertheless, although there is little evidence, DNA methylation has been reported to have a relationship with splice sites. CpG dinucleotides are distributed nonran- domly in the genome. Exon skipping and mutually exclusive exons have significantly lower levels of both CG and MCG in the exonic regions, whereas intron retention has significantly higher levels of CG in both exonic and intronic regions [34]. A DNA-binding protein, CCCTC-binding factor (CTCF), was inhibited by a methylation event of CD45 exon 5 [39]. These epigenetic features are strongly associated with alternative splicing. Furthermore, these mechanisms are known to be changed according to cell type and disease states. Especially, in cancer, the epigenetic regulation of chromatin structure effects aberrant gene expression by alternative splicing in cancer $[1,7,8]$.

Taken together, gene expression via alternative splicing is altered by complicated and mutual mechanisms, from genetic to epigenetic regulation. Therefore, it casts light on the understanding of cancer mechanisms by an investigation of alternative splicing patterns.

\section{Alternative Splicing in Cancer}

A number of studies have been reported that alternative splicing is related closely to development, cellular stress, and various diseases, including cancer, as a crucial contributor to transcriptome and proteome diversity $[1,7,8,14]$. In cancer, with increasing genomic instability, sequence substitution and aberrant alternative splicing occur frequently, leading to erroneous and dysfunctional proteins [14]. Protein isoforms made from this process are developmentally regulated and preferentially re-expressed in cancer and help the differentiation and survival of cancer cells (Fig. 2). The development

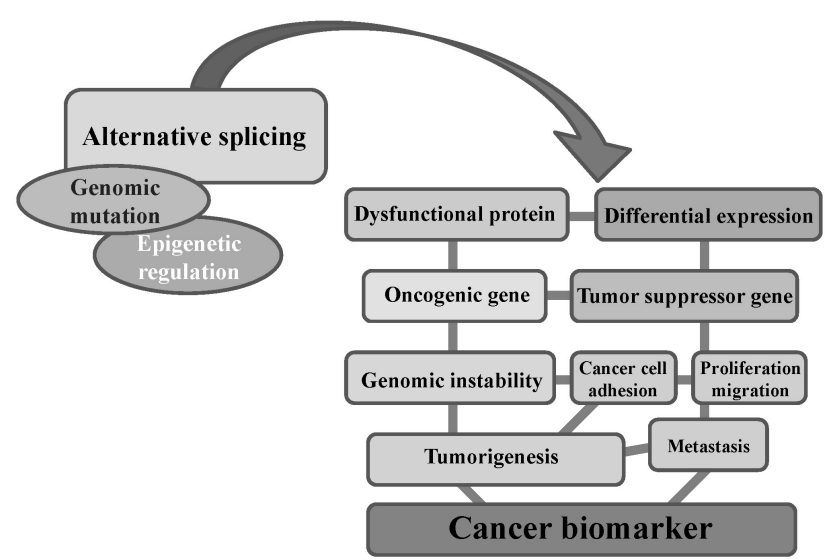

Fig. 2. Roles of alternative splicing in cancer. Dysfunctional proteins are generated by alternative splicing in cancer-related genes (oncogenic and tumor suppressor genes), increasing genomic instability. These aberrant proteins help cancerous cells to promote their growth, proliferation, and metastasis continuously (tumorigenesis). Variants of cancer-related genes expressed specifically or differently with their crucial roles for tumorigenesis could be potential biomarkers to distinguish between normal and cancer cells. 
of genomewide analysis allows large-scale examination of the relationship between alternative splicing and tumorigenesis [40, 41]. Every case of alternative splicing is reported in cancer; among these, the most frequent case is the mutually exclusive exon [14]. For example, overexpression of CD44, involved in cell proliferation, differentiation, migration, and alternative splicing, by different splice sites of CD44 during tumorigenesis indicates that it could play roles in tumor cell invasion and metastasis [42-45]. Tumor suppressor genes, such as p53 and PTEN (Phosphatase and Tensin homolog, deleted on chromosome TEN), have splicing variants associated to cancer [46]. p53 protein isoforms through alternative splicing have critical roles in many biological processes, indicating that dysregulation effects tumorigenesis [47]. Different expression of PTEN and its alternatively spliced transcripts are found to vary in different tissue types. PTEN regulates p53 stability and in turn regulates its own transcriptional activity. The PTEN splice variants retained in intron 3 region and intron 5 region have been found in breast cancer [46]. In the case of the APC gene, aberrant splice skipping of exon 4 , created by insertion of $\mathrm{T}$ in intron 4, is involved in colon cancer [48]. An alternative 5 ' splice site in $B C L-X$ results in 2 isoforms, long and short (Bcl-x (L), Bcl-x (X)), which have contrasting functions related to apoptosis and are overexpressed in various tumors $[49,50]$. Although specific roles of vascular endothelial growth factor (VEGF) isoforms are not known exactly, among the isoforms of VEGF4 involved in formation of new vessels, VEGF165 and VEGF165b, created by an alternative 3' splice site, have different expression in cancer $[51,52]$. Over 40 different MDM2 transcripts are identified in normal and cancer by alternative 5 ' and 3 ' splice sites. They mostly lose the p53 binding domain and promote tumor progression and affect prognosis independently [53, 54]. Increasing number of reports have demonstrated the expression of aberrant and abnormal splice variants in cancer cells or tissues. However, it is not enough evidence for a functional relationship between alternative splicing and cancer. Hallmarks of the initiation and early growth of cancers during tumor progression using large-scale analyses of splicing variants in the cancer EST database are needed as new RNA prognosis markers.

\section{Alternative Spliced Transcripts as Cancer Biomarkers}

Cancer is an uncontrolled state and irregularly altered genetically and epigenetically compared to normal regula-

Table 1. Alternative spliced transcript related to cancer

\begin{tabular}{|c|c|c|c|c|}
\hline Genes & Splice variants & Cancer types & Function & References \\
\hline$C D 44$ & Inclusion of exon & Various cancer & $\begin{array}{l}\text { Tumor development and } \\
\text { metastasis }\end{array}$ & Stickeler et al. [42] \\
\hline p53 & Retained intron & Breast cancer & Tumorigenesis & Okumura et al. [46] \\
\hline$p 73$ & Inclusion of exon 13 & Prostate cancer & $\begin{array}{l}\text { Prostate hyperplasia and } \\
\text { malignancy }\end{array}$ & Arvanitis et al. [55] \\
\hline PTEN & Retained intron 3 and 5 & Breast cancer & $\begin{array}{l}\text { Pathogenesis of sporadic } \\
\text { breast cancers with p53 }\end{array}$ & Okumura et al. [46] \\
\hline$A P C$ & Skipping of exon 4 & Colon cancer & $\begin{array}{l}\text { Cause of familial adenomatous } \\
\text { polyposis (FAP) }\end{array}$ & Neklason et al. [48] \\
\hline$B C L-X$ & Alternative 5 ' splice site & Hepatocellular carcinoma & Regulation of apoptosis & Takehara et al. [50] \\
\hline VEGF4 & Alternative 3 ' splice site & Various cancer & $\begin{array}{l}\text { Angiogenesis related to tumor } \\
\text { progression }\end{array}$ & Pritchard-Jones et al. [52] \\
\hline MDM2 & Alternative $5^{\prime}$ and $3^{\prime}$ splice site & Various cancer & Promote tumor progression & Bartel et al. [54] \\
\hline$B R C A 1$ & Skipping of exon 18 & Breast cancer & Breast cancer susceptibility & Okumura et al. [46] \\
\hline TACC1 & Inclusion of exon & Gastic cancer & Centrosome malfunction & Line et al. [56] \\
\hline TERT & Alternative 3' splice site & Astrocytic gliomas & Loss of telomerase activity & Kotoula et al. [57] \\
\hline KLF6 & Alternative 5 ' splice site & Prostate cancer & Tumor cell proliferation & DiFeo et al. [58] \\
\hline SURVIVIN & Retained intron & Various cancer & Tumorigenesis & Li [59] \\
\hline ASIP & Alternative 3 ' splice site & Hepatocellular carcinoma & $\begin{array}{l}\text { Enhacement of Fas-mediated } \\
\text { apoptosis }\end{array}$ & Hu et al. [60] \\
\hline NF1 & Alternative $5^{\prime}$ and $3^{\prime}$ splice site & Neurofibromatosis type I & $\begin{array}{l}\text { Inactivation of tumor } \\
\text { suppression }\end{array}$ & Barron and Lou [61] \\
\hline Caspase 8 & Retained intron & Breast cancer & Inhibitor of the caspase & Mohr et al. [62] \\
\hline $\mathrm{CDH} 17$ & Exclusion of exon 13 & Hepatocellular carcinoma & Incidence of tumor recurrence & Wang et al. [63] \\
\hline Ron & Skipping of exon 11 & Colon cancer & $\begin{array}{l}\text { Increasing of motility to } \\
\text { expressing cells }\end{array}$ & Ghigna et al. [31] \\
\hline
\end{tabular}


tion (Fig. 2). For this reason, many studies have made an effort to identify specific features and regulation mechanims of various diseases, including cancer. It is important and necessary to identify cancer markers to be able to distinguish between cancer and normal cells. Cancer markers could be very helpful in understanding tumorigenesis and developing tumor targets for therapeutic intervention. However, there still remain unsolved problems in spite of a number of studies $[7,14,45,64]$. From this point of view, alternatively spliced transcripts by mutation and cryptic splice site altered expression levels in cancer have emerged as strong candidates of cancer biomarker at the mRNA and protein level [7]. As written previously, some alternative transcripts of several genes, CD44, p53, PTEN, BCL-X, VEGF4, and MDM2, have been discovered (see also Table 1) [43, 46, 49, 50, 52, 54]. They are also associated with various cancers directly or indirectly. Recently, the discovery of biomarkers has improved by genomewide analysis $[13,65,66]$. It could be expected to provide valuable information for the association between alternative splice variants and cancers. Thus, novel candidate variants could contribute to the development of diagnostic, prognostic, and therapeutic markers.

\section{Conclusion}

RNA splicing is a core mechanism to generate mature mRNA for translation, and alternative splicing is an indispensable mechanism, stratgically leading to protein diversity for complicated regulation in eukaryotes. In cancer, alternative splicing is more flexible, leading various proteins with aberrant functions to promote growth and the spread of cancer cells. It is important to identify alternative transcripts that function specifically in cancer. These alternative transcripts could be used not only as diagnostic biomarkers but also prognostic and theraputic biomarkers. Therefore, studies on genetic and epigenetic regulation in relation to alternative splice variants in cancer could open new windows of research in answering unsolved questions of tumorigenesis.

\section{Acknowledgments}

This work was supported by a 2-Year Research Grant of Pusan National University.

\section{References}

1. Pajares MJ, Ezponda T, Catena R, Calvo A, Pio R, Montuenga LM. Alternative splicing: an emerging topic in molecular and clinical oncology. Lancet Oncol 2007;8:349-357.

2. Wahl MC, Will CL, Lührmann R. The spliceosome: design principles of a dynamic RNP machine. Cell 2009;136:701-718.
3. Matlin AJ, Clark F, Smith CW. Understanding alternative splicing: towards a cellular code. Nat Rev Mol Cell Biol 2005; 6:386-398.

4. Nilsen TW, Graveley BR. Expansion of the eukaryotic proteome by alternative splicing. Nature 2010;463:457-463.

5. Wang ET, Sandberg R, Luo S, Khrebtukova I, Zhang L, Mayr C, et al. Alternative isoform regulation in human tissue transcriptomes. Nature 2008;456:470-476.

6. Pan Q, Shai O, Lee LJ, Frey BJ, Blencowe BJ. Deep surveying of alternative splicing complexity in the human transcriptome by high-throughput sequencing. Nat Genet 2008;40:1413-1415.

7. Brinkman BM. Splice variants as cancer biomarkers. Clin Biochem 2004;37:584-594.

8. Skotheim RI, Nees M. Alternative splicing in cancer: noise, functional, or systematic? Int J Biochem Cell Biol 2007;39: 1432-1449.

9. Chang YF, Imam JS, Wilkinson MF. The nonsense-mediated decay RNA surveillance pathway. Annu Rev Biochem 2007; 76:51-74.

10. Cáceres JF, Kornblihtt AR. Alternative splicing: multiple control mechanisms and involvement in human disease. Trends Genet 2002;18:186-193.

11. Cartegni L, Chew SL, Krainer AR. Listening to silence and understanding nonsense: exonic mutations that affect splicing. Nat Rev Genet 2002;3:285-298.

12. Wang J, Zhang J, Li K, Zhao W, Cui Q. SpliceDisease database: linking RNA splicing and disease. Nucleic Acids Res 2012; 40:D1055-D1059.

13. Venables JP. Unbalanced alternative splicing and its significance in cancer. Bioessays 2006;28:378-386.

14. Venables JP. Aberrant and alternative splicing in cancer. Cancer Res 2004;64:7647-7654.

15. Black DL. Mechanisms of alternative pre-messenger RNA splicing. Annu Rev Biochem 2003;72:291-336.

16. López-Bigas N, Ouzounis CA. Genome-wide identification of genes likely to be involved in human genetic disease. Nucleic Acids Res 2004;32:3108-3114.

17. López-Bigas N, Audit B, Ouzounis C, Parra G, Guigó R. Are splicing mutations the most frequent cause of hereditary disease? FEBS Lett 2005;579:1900-1903.

18. Weisschuh N, Wissinger B, Gramer E. A splice site mutation in the PAX6 gene which induces exon skipping causes autosomal dominant inherited aniridia. Mol Vis 2012;18:751-757.

19. Kurahashi H, Takami K, Oue T, Kusafuka T, Okada A, Tawa A, et al. Biallelic inactivation of the APC gene in hepatoblastoma. Cancer Res 1995;55:5007-5011.

20. Agrawal A, Hamvas A, Cole FS, Wambach JA, Wegner D, Coghill C, et al. An intronic $A B C A 3$ mutation that is responsible for respiratory disease. Pediatr Res 2012;71:633-637.

21. Jackson IJ. A reappraisal of non-consensus mRNA splice sites. Nucleic Acids Res 1991;19:3795-3798.

22. Xue J, Rask L. The unusual 5 ' splicing border GC is used in myrosinase genes of the Brassicaceae. Plant Mol Biol 1995;29: 167-171.

23. Burset M, Seledtsov IA, Solovyev VV. Analysis of canonical and non-canonical splice sites in mammalian genomes. Nucleic Acids Res 2000;28:4364-4375. 
24. Xiong F, Gao J, Li J, Liu Y, Feng G, Fang W, et al. Noncanonical and canonical splice sites: a novel mutation at the rare noncanonical splice-donor cut site (IVS4 + 1A > G) of SEDL causes variable splicing isoforms in X-linked spondyloepiphyseal dysplasia tarda. Eur J Hum Genet 2009;17:510-516.

25. Kim E, Goren A, Ast G. Insights into the connection between cancer and alternative splicing. Trends Genet 2008;24:7-10.

26. Dominissini D, Moshitch-Moshkovitz S, Amariglio N, Rechavi G. Adenosine-to-inosine RNA editing meets cancer. Carcinogenesis 2011;32:1569-1577.

27. Maniatis T, Tasic B. Alternative pre-mRNA splicing and proteome expansion in metazoans. Nature 2002;418:236-243.

28. Dreyfuss G, Matunis MJ, Piñol-Roma S, Burd CG. hnRNP proteins and the biogenesis of mRNA. Annu Rev Biochem 1993; 62:289-321.

29. Tacke R, Manley JL. Functions of SR and Tra2 proteins in pre-mRNA splicing regulation. Proc Soc Exp Biol Med 1999; 220:59-63.

30. Colapietro P, Gervasini C, Natacci F, Rossi L, Riva P, Larizza L. NF1 exon 7 skipping and sequence alterations in exonic splice enhancers (ESEs) in a neurofibromatosis 1 patient. Hum Genet 2003;113:551-554.

31. Ghigna C, Giordano S, Shen H, Benvenuto F, Castiglioni F, Comoglio PM, et al. Cell motility is controlled by SF2/ASF through alternative splicing of the Ron protooncogene. Mol Cell 2005;20:881-890.

32. Schwartz S, Meshorer E, Ast G. Chromatin organization marks exon-intron structure. Nat Struct Mol Biol 2009;16: 990-995.

33. Luco RF, Pan Q, Tominaga K, Blencowe BJ, Pereira-Smith OM, Misteli T. Regulation of alternative splicing by histone modifications. Science 2010;327:996-1000.

34. Zhou Y, Lu Y, Tian W. Epigenetic features are significantly associated with alternative splicing. BMC Genomics 2012;13: 123.

35. Andersson R, Enroth S, Rada-Iglesias A, Wadelius C, Komorowski J. Nucleosomes are well positioned in exons and carry characteristic histone modifications. Genome Res 2009;19: 1732-1741.

36. Kolasinska-Zwierz P, Down T, Latorre I, Liu T, Liu XS, Ahringer J. Differential chromatin marking of introns and expressed exons by H3K36me3. Nat Genet 2009;41:376-381.

37. Wilhelm BT, Marguerat S, Aligianni S, Codlin S, Watt S, Bähler J. Differential patterns of intronic and exonic DNA regions with respect to RNA polymerase II occupancy, nucleosome density and H3K36me3 marking in fission yeast. Genome Biol 2011;12:R82.

38. Gunderson FQ, Merkhofer EC, Johnson TL. Dynamic histone acetylation is critical for cotranscriptional spliceosome assembly and spliceosomal rearrangements. Proc Natl Acad Sci U S A 2011;108:2004-2009.

39. Shukla S, Kavak E, Gregory M, Imashimizu M, Shutinoski B, Kashlev M, et al. CTCF-promoted RNA polymerase II pausing links DNA methylation to splicing. Nature 2011;479:74-79.

40. David CJ, Manley JL. Alternative pre-mRNA splicing regulation in cancer: pathways and programs unhinged. Genes Dev 2010;24:2343-2364.
41. Venables JP, Klinck R, Koh C, Gervais-Bird J, Bramard A, Inkel $\mathrm{L}$, et al. Cancer-associated regulation of alternative splicing. Nat Struct Mol Biol 2009;16:670-676.

42. Stickeler E, Kittrell F, Medina D, Berget SM. Stage-specific changes in SR splicing factors and alternative splicing in mammary tumorigenesis. Oncogene 1999;18:3574-3582.

43. Naor D, Nedvetzki S, Golan I, Melnik L, Faitelson Y. CD44 in cancer. Crit Rev Clin Lab Sci 2002;39:527-579.

44. Faustino NA, Cooper TA. Pre-mRNA splicing and human disease. Genes Dev 2003;17:419-437.

45. Kalnina Z, Zayakin P, Silina K, Linē A. Alterations of premRNA splicing in cancer. Genes Chromosomes Cancer 2005; 42:342-357.

46. Okumura N, Yoshida H, Kitagishi Y, Nishimura Y, Matsuda S. Alternative splicings on $p 53, B R C A 1$ and PTEN genes involved in breast cancer. Biochem Biophys Res Commun 2011; 413:395-399.

47. Wei J, Zaika E, Zaika A. p53 family: role of protein isoforms in human cancer. J Nucleic Acids 2012;2012:687359.

48. Neklason DW, Solomon CH, Dalton AL, Kuwada SK, Burt RW. Intron 4 mutation in $A P C$ gene results in splice defect and attenuated FAP phenotype. Fam Cancer 2004;3:35-40.

49. Olopade OI, Adeyanju MO, Safa AR, Hagos F, Mick R, Thompson CB, et al. Overexpression of BCL-x protein in primary breast cancer is associated with high tumor grade and nodal metastases. Cancer J Sci Am 1997;3:230-237.

50. Takehara T, Liu X, Fujimoto J, Friedman SL, Takahashi H. Expression and role of Bcl-xL in human hepatocellular carcinomas. Hepatology 2001;34:55-61.

51. Woolard J, Wang WY, Bevan HS, Qiu Y, Morbidelli L, Pritchard-Jones RO, et al. VEGF165b, an inhibitory vascular endothelial growth factor splice variant: mechanism of action, in vivo effect on angiogenesis and endogenous protein expression. Cancer Res 2004;64:7822-7835.

52. Pritchard-Jones RO, Dunn DB, Qiu Y, Varey AH, Orlando A, Rigby $\mathrm{H}$, et al. Expression of $\operatorname{VEGF}(\mathrm{xxx}) \mathrm{b}$, the inhibitory isoforms of VEGF, in malignant melanoma. Br J Cancer 2007; 97:223-230.

53. Bartel F, Taubert H, Harris LC. Alternative and aberrant splicing of MDM2 mRNA in human cancer. Cancer Cell 2002; 2:9-15.

54. Bartel F, Harris LC, Wurl P, Taubert H. MDM2 and its splice variant messenger RNAs: expression in tumors and downregulation using antisense oligonucleotides. Mol Cancer Res 2004;2:29-35.

55. Arvanitis DA, Lianos E, Soulitzis N, Delakas D, Spandidos DA. Deregulation of p73 isoform equilibrium in benign prostate hyperplasia and prostate cancer. Oncol Rep 2004;12: 1131-1137.

56. Line A, Slucka Z, Stengrevics A, Li G, Rees RC. Altered splicing pattern of TACC1 mRNA in gastric cancer. Cancer Genet Cytogenet 2002;139:78-83.

57. Kotoula V, Barbanis S, Nikolakaki E, Koufoyannis D, Papadimitriou CS, Karkavelas G. Relative expression of human telomerase catalytic subunit (hTERT) transcripts in astrocytic gliomas. Acta Neuropathol 2004;107:443-451.

58. DiFeo A, Martignetti JA, Narla G. The role of KLF6 and its 
splice variants in cancer therapy. Drug Resist Updat 2009;12: 1-7.

59. Li F. Role of survivin and its splice variants in tumorigenesis. Br J Cancer 2005;92:212-216.

60. Hu Y, Fang C, Xu Y. The effect of isoforms of the cell polarity protein, human ASIP, on the cell cycle and Fas/FasL-mediated apoptosis in human hepatoma cells. Cell Mol Life Sci 2005; 62:1974-1983.

61. Barron VA, Lou H. Alternative splicing of the neurofibromatosis type I pre-mRNA. Biosci Rep 2012;32:131-138.

62. Mohr A, Zwacka RM, Jarmy G, Büneker C, Schrezenmeier H, Döhner K, et al. Caspase-8L expression protects CD34+ hematopoietic progenitor cells and leukemic cells from CD95mediated apoptosis. Oncogene 2005;24:2421-2429.
63. Wang XQ, Luk JM, Leung PP, Wong BW, Stanbridge EJ, Fan ST. Alternative mRNA splicing of liver intestine-cadherin in hepatocellular carcinoma. Clin Cancer Res 2005;11(2 Pt 1):483489.

64. Pettigrew CA, Brown MA. Pre-mRNA splicing aberrations and cancer. Front Biosci 2008;13:1090-1105.

65. Cuperlovic-Culf M, Belacel N, Culf AS, Ouellette RJ. Data analysis of alternative splicing microarrays. Drug Discov Today 2006;11:983-990.

66. Omenn GS, Yocum AK, Menon R. Alternative splice variants, a new class of protein cancer biomarker candidates: findings in pancreatic cancer and breast cancer with systems biology implications. Dis Markers 2010;28:241-251. 